\title{
Padrão da infecção pelo HIV/AIDS em Manaus, Estado do Amazonas, no período de 1986 a 2000
}

\author{
Pattern of HIV/AIDS infection in Manaus, State of Amazonas, between 1986 and 2000
}

\author{
Leila Cristina Ferreira da Silva ${ }^{1}$, Elizabeth Moreira dos Santos ${ }^{2}$, Antonio Levino da Silva Neto ${ }^{3}$, \\ Angélica Espinosa Miranda ${ }^{4}$, Sinésio Talhari ${ }^{1}$ e Luciano de Medeiros Toledo ${ }^{2}$
}

\begin{abstract}
RESUMO
O objetivo deste estudo foi descrever a infecção pelo HIV em Manaus, Amazonas no período de 1986 a 2000 . Estudo descritivo dos casos confirmados de HIV/Aids em adultos, registrados nos prontuários do Serviço de Referência Estadual, foi realizado. Para o delineamento da epidemiologia espacial e tendência histórica foram considerados os períodos: 1986-1990, 1991-1995 e 1996-2000. As variáveis comportamentais, sociais e clínicas foram analisadas por meio de estatística descritiva. Mapas temáticos apresentaram os padrões e tendências espaciais e taxas de incidência segundo bairros de residência. Entre os 1.400 casos estudados, a letalidade diminuiu de 61,3\% para 17,8\%, a razão entre sexos (4 homens/1 mulher) diminuiu durante o período do estudo, a principal via de exposição foi a sexual: bissexual (31\%) e heterossexual (19,3\%) e 0 alto índice de diagnóstico tardio realizado na fase sintomática da Aids (50,8\%). Este estudo mostrou que a infecção pelo HIV/Aids em Manaus apresenta difusão lenta e progressiva localizada na área central da cidade, dispersiva no sentido centro-sul para o norte, leste e oeste.
\end{abstract}

Palavras-chaves: Vírus da imunodeficiência humana. Síndrome da imunodeficiência adquirida. Epidemiologia. Distribuição espacial.

\begin{abstract}
The objective of this study was to describe HIV infection in Manaus, Amazonas, between 1986 and 2000. This was a descriptive study on confirmed cases of HIV/ AIDS among adults, from the medical records of the State Reference Service. To delineate the spatial epidemiological profile and historical trends, the following periods were considered: 1986-1990, 1991-1995 and 1996-2000. The behavioral, social and clinical variables were analyzed by means of descriptive statistics. The spatial trends and patterns and the incidence rates were presented according to residential district using thematic maps. Among the 1,400 cases studied, the mortality rate decreased from $61.3 \%$ to $17.8 \%$ and the gender ratio (four men/one woman) decreased over the study period. The main exposure route was sexual: bisexual (31\%) and heterosexual (19.3\%). There was a high rate of late diagnosis, made during the symptomatic phase of AIDS (50.8\%). This study showed that HIV/AIDS infection in Manaus has spread slowly and progressively from the central area of the city towards the south, north, east and west.
\end{abstract}

Key-words: Human immunodeficiency virus. Acquired immunodeficiency syndrome. Epidemiology. Spatial distribution.

A partir de 1990, constatou-se transição no perfil epidemiológico da epidemia brasileira da síndrome de imunodeficiência adquirida (AIDS), apresentando tendência crescente de pauperização, heterossexualização, feminização e interiorizaçã $0^{3}{ }^{19}$. As desigualdades da sociedade brasileira favorecem a difusão da infecção pelo vírus da imunodeficiência humana (HIV) no país, revelando uma epidemia de múltiplas dimensões que vem, ao longo do tempo, sofrendo transformações significativas em seu perfil epidemiológico ${ }^{14}$. A forma de organização social do espaço e as desigualdades econômicas e culturais, presentes no interior da estrutura da sociedade brasileira, são os principais fatores que têm determinado e

1. Gerência de Dermatologia e DST/Aids, Fundação de Medicina Tropical do Amazonas, Manaus, AM. 2. Escola Nacional de Saúde Pública, Fundação Osvaldo Cruz, Rio de Janeiro, RJ. 3. Centro de Pesquisa Leônidas e Maia Deâne, Fundação Osvaldo Cruz na Amazônia, Manaus, AM. 4. Programa de Pós-Graduação em Doenças Infecciosas, Núcleo de Doenças Infecciosas, Universidade Federal do Espírito Santo, Vitória, ES. Endereço para correspondência: MsC. Leila Cristina Ferreira da Silva. Fundação de Medicina Tropical do Amazonas/FMT. Av. Pedro Teixeira 25, Dom Pedro I, 69040-000 Manaus, AM.

Tel: 5592 2127-3459

e-mail: leilac@fmt.am.gov.br

Recebido para publicação em 20/03/2009

Aceito em 15/09/2009 condicionado a receptividade e a vulnerabilidade à manutenção e difusão da epidemia de diversas doenças transmissíveis no país ${ }^{15}$. Muitas dessas doenças têm atingido áreas de grande isolamento geográfico, como aquelas da Região Amazônica ${ }^{15}$.

Sob o ponto de vista sanitário, a difusão da infecção pelo HIV/Aids na região norte do Brasil e no Estado do Amazonas, em particular, torna-se especialmente preocupante, sobretudo pela situação de grande vulnerabilidade deste espaço geográfico e que é determinada pela diversidade étnico-cultural de seus grupos populacionais $^{32}$. As condições precárias de vida da população, carentes de acesso às necessidades básicas e de assistência à saúde, face à magnitude da doença e tendência epidemiológica atuais, principalmente a respeito da interiorização $0^{314}$ e possibilidade de maior relevância entre os índios, acentua a importância da informação e conhecimento do processo epidêmico de difusão da infecção pelo HIV/Aids no Amazonas.

No Estado do Amazonas, as notificações dos casos retratam maior concentração da doença em Manaus (capital), que concentra aproximadamente $90 \%$ dos $\operatorname{casos}^{8}$. Este dado pode ser considerado um indicador de desigualdade na distribuição geográfica no interior deste estado ${ }^{8}$. A vulnerabilidade espacial da Cidade de Manaus, e seu favorecimento à concentração dos casos 
de infecção pelo HIV/Aids em algumas de suas áreas urbanas, não estão ainda bem esclarecidos. Sua dinâmica urbana reflete a realidade regional, historicamente submetida a uma variedade de eventos socioeconômicos de maior ou menor duração e/ou impacto e, assim, conformando sua paisagem que tem aos arredores a floresta amazônica ${ }^{4}$.

O espaço social de Manaus encontra-se em constante processo de urbanização, expandindo-se em suas áreas periféricas, principalmente pelo êxodo do contingente populacional residente no interior do estado em direção à capital em busca de melhores condições materiais de vida, incluindo-se acesso à tecnologia $\mathrm{e}$ à modernidade ${ }^{2}$. Manaus apresenta grande diversidade devido a sua origem sóciocultural indígena e forte influência de migração proveniente da região Nordeste do Brasil, desde o seu período de colonização e ciclos econômicos extrativistas exploratórios, de projetos de povoamento das áreas florestais e de pólo industrial brasileiro ${ }^{4}$.

Esse artigo tem como objetivo descrever o perfil epidemiológico da infecção pelo HIV/Aids na Cidade de Manaus, pois o conhecimento sobre a peculiaridade de comportamento da epidemia, em relação aos fatores determinantes e condicionantes de vulnerabilidade da população, é fundamental para melhor consistência e efetividade de suas ações de prevenção e controle.

\section{MATERIAL E MÉTODOS}

Foi realizado estudo descritivo que avaliou os casos de infecção pelo HIV e de Aids, com 13 anos de idade ou mais, residentes em Manaus, que apresentavam evidência comprovada de infecção pelo HIV segundo as normas do Ministério da Saúde $(\mathrm{MS})^{16}$, no período compreendido entre 1 de janeiro de $1986 \mathrm{e}$ 31 de dezembro de 2000.

Seleção da amostra. Os casos foram selecionados a partir de informações de prontuários do único Serviço de Assistência Especializada (SAE) existente no Estado do Amazonas, na Fundação de Medicina Tropical do Amazonas (FMT-AM).

A revisão na definição de caso de Aids no Brasil em indivíduo com 13 anos ou mais de idade utilizada neste estudo ocorreu no ano de 1998. Esta definição do MS inclui referências clínicas e laboratoriais específicas e adiciona os seguintes critérios definidores de $\operatorname{casos}^{16}$ : Critério do Center Disease Control (CDC) modificado; Critério Rio de Janeiro-Caracas; Critério excepcional óbito; Critério excepcional Aids Related Complex (ARC) junto ao óbito.

Definição das variáveis. A vulnerabilidade sexual foi definida pela presença de variáveis comportamentais relacionadas às possíveis exposições sexuais ao HIV. A variável sintomas clínicos foi definida a partir dos sintomas apresentados na primeira consulta. A regularidade ao tratamento foi classificada em três categorias a partir da data da primeira consulta, sendo: regular, para aqueles com três ou mais comparecimentos anuais para atendimentos; irregular para aqueles casos com comparecimento menor que três vezes ao ano; abandono para aqueles sem comparecimento, no período de um ano ou mais. Neste estudo, foram, também, incluídos os casos diagnosticados no momento do óbito, ainda na primeira consulta.

Estudo piloto. Precedendo a coleta de dados foi realizado um estudo piloto em uma amostra aleatória de prontuários a fim de se avaliar a adequação do instrumento de pesquisa e apontar possíveis estratégias de composição.

Coleta de dados. Para o delineamento do padrão epidemiológico e das tendências da infecção pelo HIV/ Aids, no espaço geográfico de Manaus, foram considerados períodos distintos de estudo na série histórica de 1986 a 2000, compreendendo: 1986 a 1990, 1991 a 1995 e 1996 a 2000 .

O banco de dados foi construído com base nas informações dos registros de casos de infecção pelo HIV/Aids a partir dos prontuários existentes no SAE/FMT-AM. Foram incluídas no instrumento de pesquisa variáveis presentes na ficha de notificação de casos de Aids do Sistema Nacional de Agravos Notificáveis (SINAN) e variáveis disponíveis nos prontuários dos casos estudados.

Análise de dados. Os casos foram analisados utilizandose técnicas de estatística descritiva. Os dados foram tabulados e distribuídos por grupos etários, gênero, situação marital, companheiro infectado pelo HIV, tipo de exposição ao HIV, vulnerabilidade sexual, história de doenças sexualmente transmissíveis (DST), forma clínica, sintomas clínicos, regularidade ao tratamento e ocupação profissional. Os aplicativos utilizados foram o Excel e Epiinfo 6.0.

A análise de tendência foi realizada através de modelo de regressão polinomial, sendo a variável dependente a taxa de incidência por 100.000 habitantes (Y) e a variável independente, 0 ano de diagnóstico dos casos estudados (X), ambas consideradas para 0 município de Manaus ${ }^{1}{ }^{10}$. Foram calculadas as taxas de incidência de casos de infecção pelo HIV/Aids, por ano de diagnóstico e taxas de incidência médias, por períodos analisados.

Inicialmente, um diagrama de dispersão foi elaborado, com o auxílio do aplicativo Excel, para correlacionar as taxas de incidência com os anos de diagnósticos em estudo. Posteriormente, utilizando-se o mesmo aplicativo, identificouse a linha de tendência melhor ajustada segundo coeficiente de determinação $\left(\mathrm{R}^{2}\right)$ com maior poder explicativo, curva de regressão polinomial de segundo grau $\left(\mathrm{Y}=\mathrm{b}_{0}+\mathrm{b}_{1} \mathrm{X}+\mathrm{b}_{2} \mathrm{X}^{2}\right)^{1}$.

Mapas temáticos foram construídos no aplicativo ArcGis 8.3, nos quais foram apresentados os padrões de difusão e tendências de distribuição espacial de casos e das variações de taxas de incidência, por 10.000 habitantes (para adequar ao menor número populacional), segundo bairros de residência dos casos registrados na Cidade de Manaus ${ }^{10}$.

Aspectos éticos. Este estudo foi realizado de acordo com a resolução 196/96 do Conselho Nacional de Saúde e submetido e aprovado pela Comissão de Ética em Pesquisa da Escola Nacional de Saúde Pública, Fundação Osvaldo Cruz, Rio de Janeiro (Protocolos n $\left.n^{0} .71 / 2002\right)$ e da FMT-AM (n⿳⺈ 1236/2002). 


\section{RESULTADOS}

Nesta série histórica, realizada em Manaus, com dados de 1986 a 2000, foram oficialmente registrados 1.400 casos de infecção pelo HIV/Aids. Esses casos ocorreram em 55 (98,2\%) dos 56 bairros existentes e em todas as seis zonas administrativas (norte, sul, leste, oeste, centro sul e centro oeste).

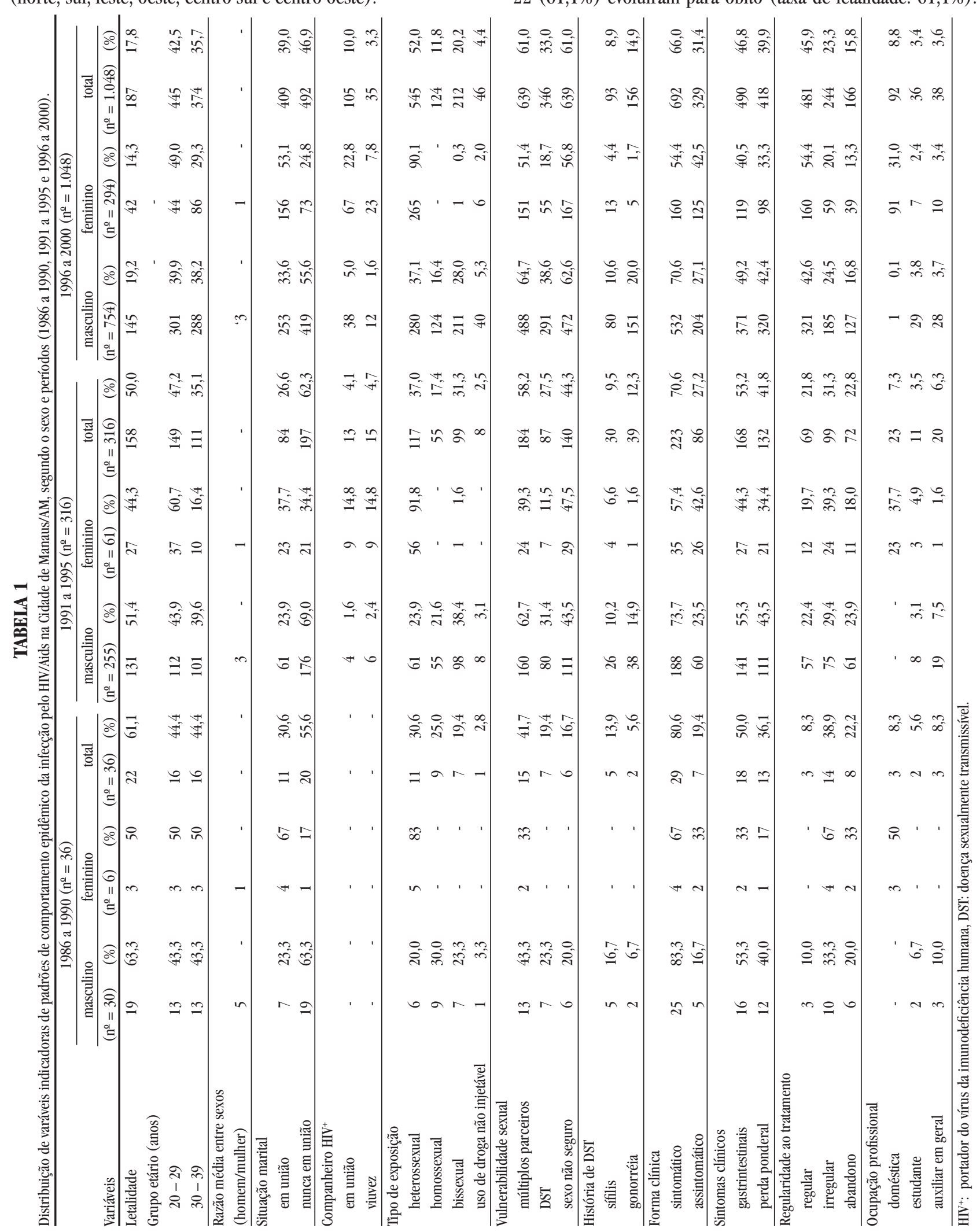

A Tabela 1 apresenta os resultados que se destacaram nos períodos estudados, 1986-1990, 1991-1995 e 1996-2000, quanto ao padrão epidemiológico dos casos de infecção pelo HIV/Aids em Manaus. 0 primeiro caso de Aids registrado em Manaus foi detectado em 1986.

No primeiro período (1986-1990), dos 36 casos registrados, $22(61,1 \%)$ evoluíram para óbito (taxa de letalidade: 61,1\%). 
A razão entre os sexos foi de 5 homens/1 mulher. 0 tipo de exposição heterossexual destacou-se com $11(30,6 \%)$ casos e os tipos de exposição homossexual e bissexual totalizaram, respectivamente, nove (25\%) e sete $(19,4 \%)$ casos. Quanto ao uso de drogas injetáveis (UDI), houve um $(2,8 \%)$ caso e não injetáveis (UDNI), dois $(6,7 \%)$ casos. Quanto à vulnerabilidade sexual destacou-se história de múltiplos parceiros com 15 (41,7\%) casos. A ausência de informações quanto à história de DST foi expressiva; dentre os casos com história de DST, observou-se maior frequiência da sífilis, com cinco $(16,7 \%)$ casos.

A detecção dos casos na forma clínica sintomática da Aids ocorreu em 29 (80,6\%) casos; os principais sintomas clínicos apresentados foram gastrenterites associadas à perda ponderal, em $18(50 \%)$ e $13(36,1 \%)$ casos, respectivamente. 0 relato de tratamento irregular foi encontrado em $14(38,9 \%)$ casos e 0 abandono do tratamento em oito $(22,2 \%)$ casos. Os casos de óbito detectados no momento da primeira consulta foram 11 (30,6\%), todos do sexo masculino.

No segundo período do estudo (1991 a 1995), conforme se pode observar ainda na Tabela $\mathbf{1}$, foram registrados 316 novos casos de infecção pelo HIV/AIDS, um acréscimo de 7,8 vezes no número de casos em relação ao período anterior. Ao final desse período, 158 casos evoluíram para óbito (taxa de letalidade de 50\%). A razão de sexo foi em média 4 homens para cada mulher. A principal categoria de exposição ao HIV foi a sexual $271(87,7 \%)$ casos. A exposição ao UDNI ocorreu em oito $(2,5 \%)$ casos e por transfusão sanguínea em quatro $(1,3 \%)$ casos. Quanto à vulnerabilidade sexual, destacaram-se os registros de $160(62,7 \%)$ casos com história de múltiplos parceiros para o sexo masculino e de 29 (47,5\%) de sexo não seguro, para 0 sexo feminino. Dentre todos os casos registrados, $69(21,8 \%)$ referiram história pregressa de DST. Um total de 223 (70,6\%) casos era sintomático. As manifestações mais frequentes foram gastrenterites, com 168 (53,2\%) casos, seguidas da perda ponderal, com 134 (42,4\%). Nesse período, destacou-se, em ordem decrescente, a irregularidade de tratamento, em 99 $(31,3 \%)$ casos e de abandono com $72(22,8 \%)$ casos. Foram detectados $76(24,1 \%)$ casos de óbito na primeira consulta.

No terceiro e último período do estudo (1996 a 2000), também descrito na Tabela 1, foram registrados 1.048 novos casos de infecção pelo HIV/Aids, aumento de 231,6\% em relação aos cinco anos anteriores. Nesse período, foram contabilizados 187 óbitos (taxa de letalidade de 17,8 \%). A razão entre os sexos foi de 3 homens para cada mulher. No período, predominaram os casos oriundos de exposição sexual, 947 (90,4\%) casos; o UDNI totalizou $46(4,4 \%)$ casos e a transfusão sanguínea representou $1,1 \%$ dos casos. Quanto à vulnerabilidade sexual, destacou-se a multiplicidade de parceria sexual e o sexo não seguro. As DST foram mais frequentes no sexo masculino, com 231 (30,6\%) casos. No mesmo período, foram registrados três $(0,3 \%)$ casos de infecção pelo HIV/Aids em profissionais do sexo.

Dos casos registrados no terceiro período, 692 (66\%) encontravam-se na fase sintomática e as manifestações clínicas mais frequentes foram as gastrintestinais e a perda ponderal. Destacaram-se, em ordem decrescente, 244 (23,3\%) com irregularidade de tratamento; o abandono ultrapassou os 15\%; os casos de óbito ocorridos no momento da primeira consulta foram $141(13,4 \%)$.

A Figura 1 apresenta os achados relacionados às tendências da infecção pelo HIV/Aids na Cidade de Manaus, nos mesmos períodos estudados. A observação, por período, da série histórica demonstrou comportamento similar à tendência histórica total (1986 a 2000), com linha de tendência polinomial apresentando $\mathrm{R} 2=0,9623(\mathrm{y}=0,0863 \mathrm{x} 2+0,1649 \mathrm{x}-0,6885)$ e taxa de incidência média de 7,8 por 100.000 habitantes.

A média da taxa de incidência de Aids no primeiro período (1986 a 1990) foi de 0,76 casos por 100.000 habitantes. Evidenciou-se crescimento lento e progressivo na curva. No

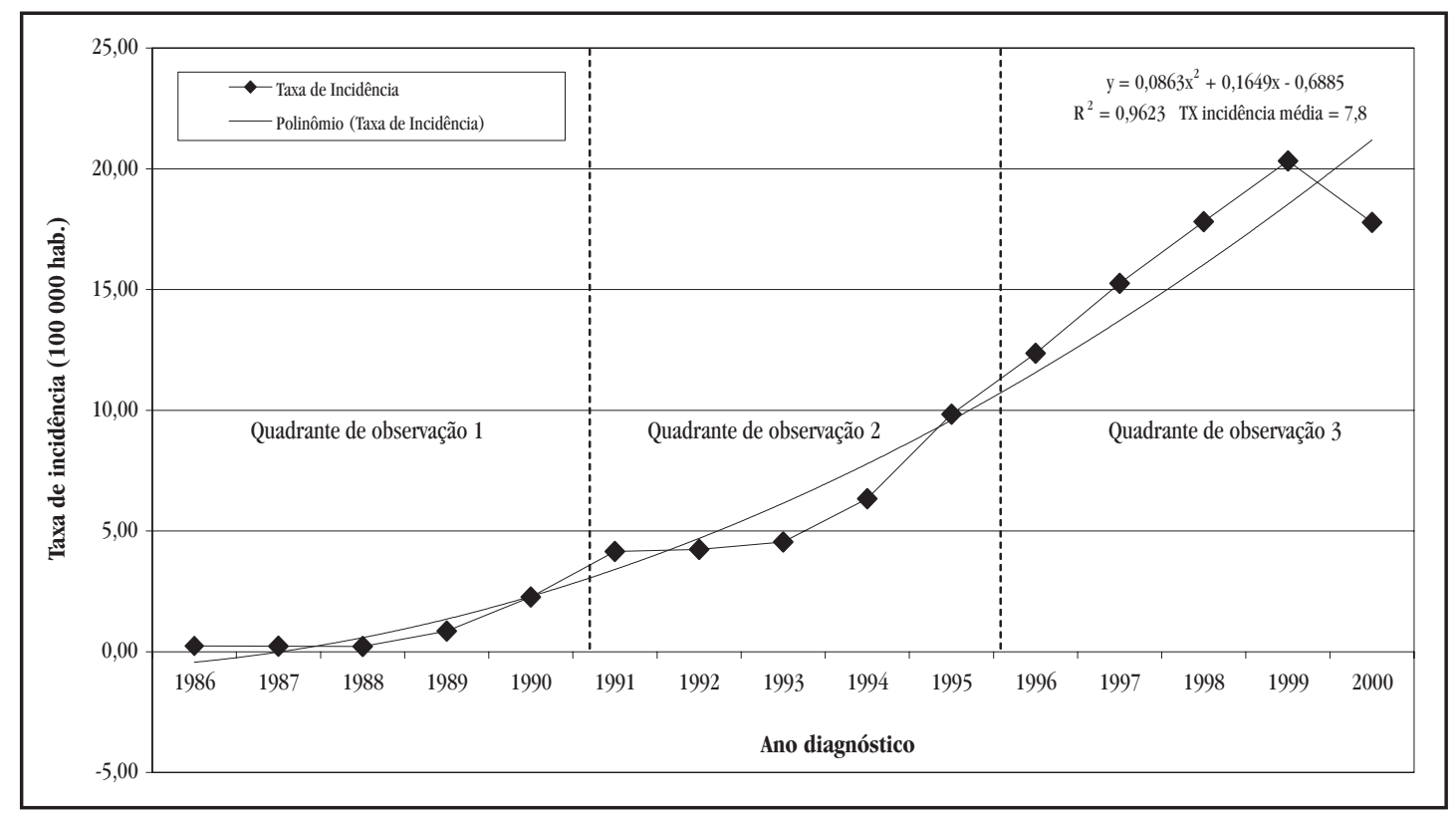

FIGURA 1

Distrtibuição da taxa de incidência da infecção pelo HIV/aids na Cidade de Manaus, segundo ano de diagnóstico, no período de 1986 a 2000. 
segundo período (1991 a 1995), houve maior grau de aceleração e de expansão da epidemia. A taxa de incidência média alcançou 5,82 , cifra 7,5 vezes maior do que a verificada no período anterior. No último período de estudo (1996 a 2000) houve incremento significativo das taxas de incidência, por 100.000 habitantes, até 1999, quando alcançou 20,32 e redução pontual em 2000, para 17,78 .

Os padrões espaciais dos casos de infecção pelo HIV/Aids nos períodos de 1986 a 1990, de 1991 a 1995 e de 1996 a 2000, encontram-se apresentados nas Figuras 2,3 e 4, de acordo com os bairros da Cidade de Manaus.

Na Figura 2, observa-se que, no período de 1986 a 1990, a difusão espaço-temporal inicial da infecção se deu em torno da área central da cidade, englobando, ainda, áreas adjacentes, no sentido sul-norte, com taxas que variavam, em nível de bairros, entre 0,33 a 5,38 por 10.000 habitantes. No segundo período de estudo, 1991 a 1995 (Figura 3), os casos de infecção pelo HIV/Aids distribuíram-se em 47 (83,9\%) dos 56 bairros da Cidade de Manaus. As taxas de incidência nesse período foram maiores, sobretudo nos bairros do Centro $(14,78)$.

No último período (1996 a 2000), com exceção de dois, todos os outros 54 bairros da Cidade de Manaus apresentaram casos de infecção pelo HIV/Aids. A expansão epidêmica intensificou-se na área centro-sul, centro-oeste, centro-norte e centro-leste, principalmente para o norte (Figura 4).

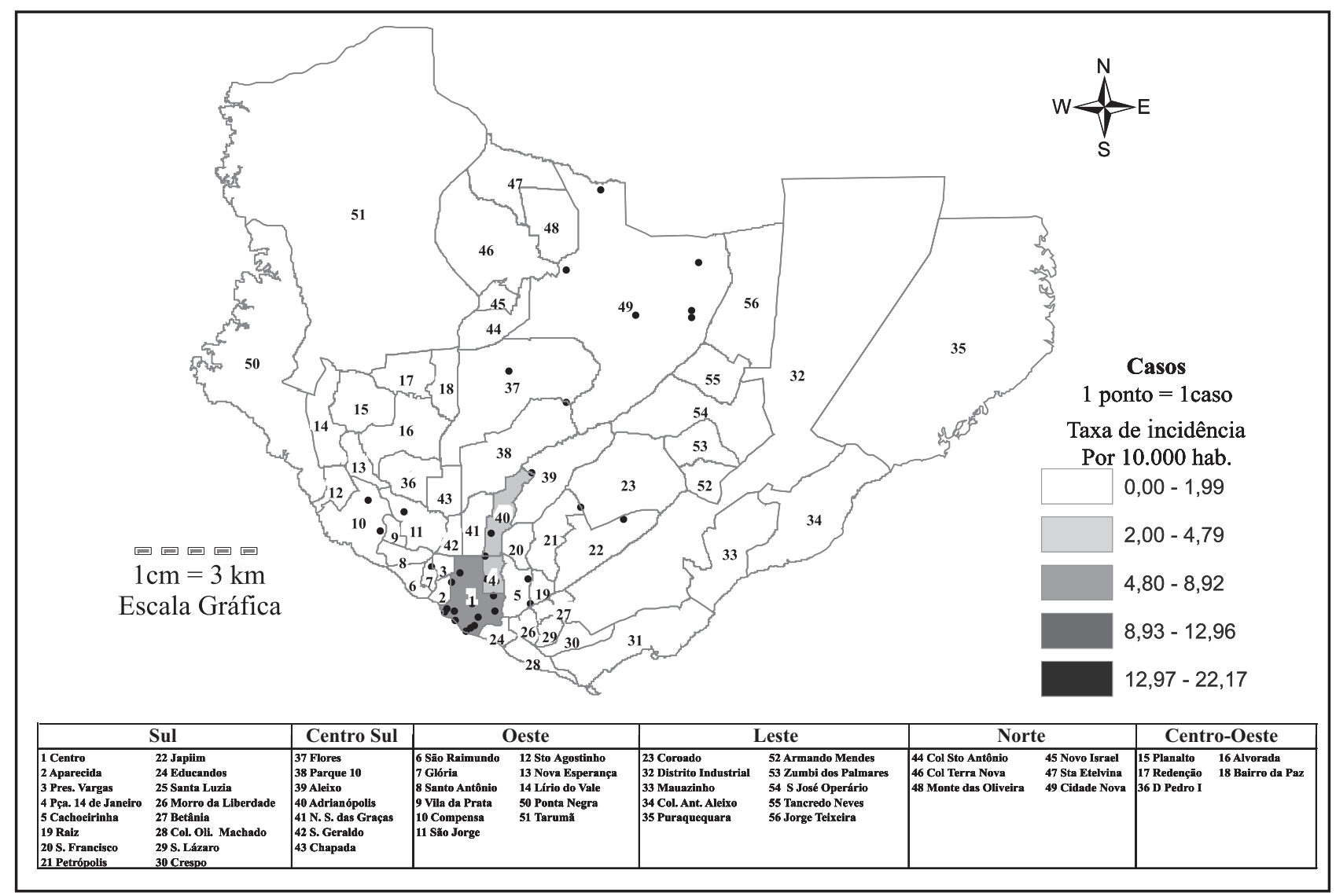

FIGURA 2

Distribuição de casos e taxas de incidência de infecção pelo HIV/AIDS segundo os bairros da Cidade de Manaus, no período de 1986 a 1990. 


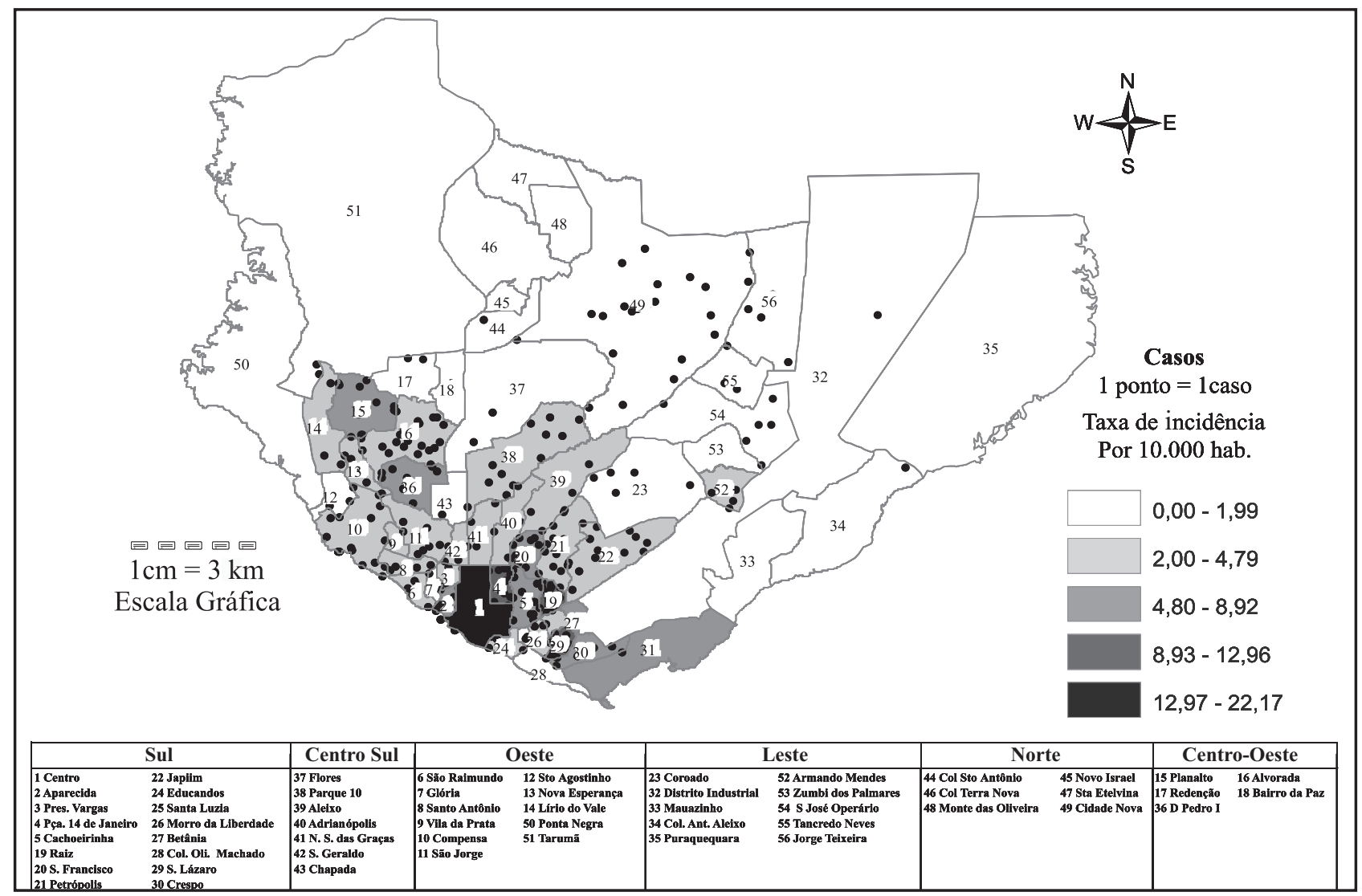

FIGURA 3

Distribuição de casos e taxas de incidência de infecção pelo HIV/AIDS segundo os bairros da Cidade de Manaus, no período de 1991 a 1995.

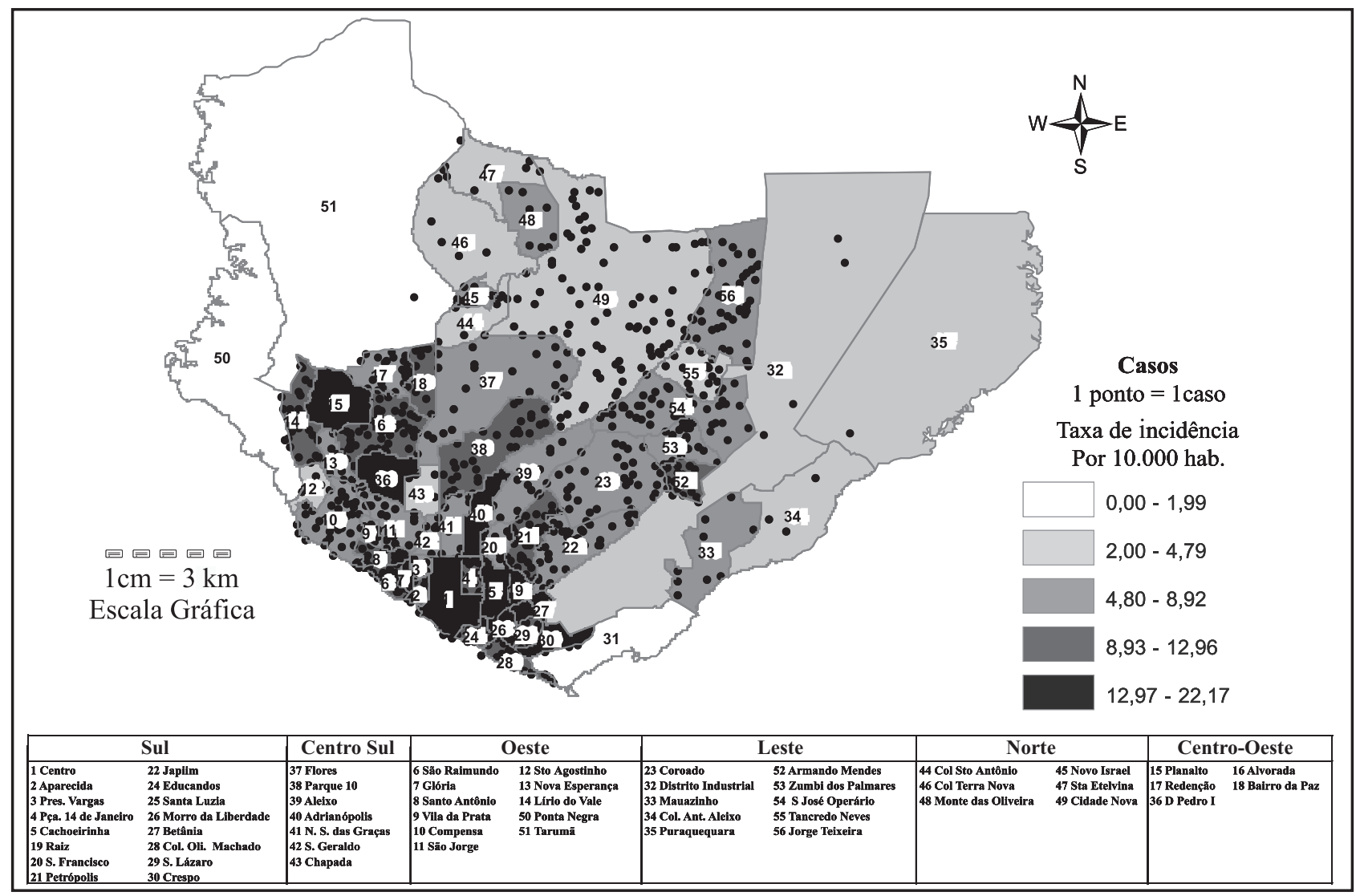

FIGURA 4

Distribuição de casos e taxas de incidência de infecção pelo HIV/AIDS segundo os bairros da Cidade de Manaus, no período de 1996 a 2000. 


\section{DISCUSSÃo}

No presente estudo, foi analisado o padrão epidemiológico da infecção pelo HIV/Aids na cidade de Manaus no período de 1986 a 2000. 0 estudo demonstrou, sob o ponto de vista evolutivo, padrões temporais e espaciais bastante diversos, embora predominando, em termos gerais, um padrão lento e progressivo de difusão. Estudos que tentam conhecer a dinâmica da epidemia pelo HIV/ Aids são importantes tanto para o diagnóstico das tendências da epidemia, como à proposição de estratégias preventivas sensíveis às particularidades regionais e às características socioeconômicas e culturais de diferentes segmentos populacionais, habitualmente relegadas a plano secundário nos estudos cuja base de análise é constituída exclusivamente por dados relativos aos indivíduos singulares ${ }^{12}$.

O padrão lento de difusão observado pode ser explicado, em parte, pelo relativo isolamento geográfico dessa Capital o que dificulta as relações interpessoais de suas populações com 0 mundo externo e vice-versa ${ }^{18}$. Esta situação seguramente tem contribuído para menor pressão e circulação viral sobre o espaço da cidade. Ao término do período estudado, a transmissão do HIV/Aids já atingia praticamente todos os espaços socialmente ocupados dessa Capital, independentemente de heterogeneidades existentes no interior de seus distintos bairros.

Os resultados obtidos permitiram identificar que a evolução temporal da infecção pelo HIV/Aids na Cidade de Manaus, apresentou três padrões relativamente distintos. 0 padrão observado no primeiro período (1986 a 1990) foi caracterizado por progressão lenta da epidemia. 0 padrão da epidemia no período seguinte (1991 a 1995) expressou-se por crescimento moderado, com aumento relativo do número de casos e na intensidade da transmissão, ampliação significativa dos espaços sociais de ocorrência da infecção. E no último período de estudo (1996 a 2000), o padrão da infecção pelo HIV/Aids na Cidade de Manaus caracterizou-se por forte incremento da epidemia, com um aumento expressivo do número de casos e das taxas de incidência, com grande ampliação dos espaços sociais de ocorrência da infecção. A partir dos resultados, foi possível constatar que a epidemia pelo HIV/Aids em Manaus experimentou de forma mais lenta as mesmas modificações já descritas para todo o país ${ }^{619}$.

A natureza da ocupação profissional, servindo como aproximação das condições socioeconômicas dos casos HIV/Aids, demonstrou que em Manaus a epidemia atingiu, principalmente, os indivíduos com menor qualificação profissional e menor escolaridade. Grupo socioeconômico e culturalmente mais vulnerável à infecção pelo HIV. Determinadas ocupações se mostram particularmente vulneráveis à difusão da epidemia, provavelmente, pelo fato de refletirem estilos de vida e opções pessoais, e, simultaneamente, definir redes sociais de interação com características específicas ${ }^{7}$.

Distintamente do comportamento encontrado na Cidade de Manaus, no Brasil houve redução mais significativa na aproximação da razão média entre os sexos entre os casos de Aids no país, a partir do período de 1991 a $1995^{17}$. Outra distinção refere-se à participação da exposição homo/bisssexual que foi importante em
1984, no Brasil, com redução progressiva na década posterior ${ }^{13}$. O Brasil, desde a década de 1980, já apresentava tendência de heterossexualização, superando as categorias homo/bissexual a partir de $1992^{13}$. Em Manaus, somente a partir dos meados dos anos 90, a exposição heterossexual foi forma mais relevante de transmissão do HIV/Aids. O contato heterossexual resulta em crescimento substancial dos casos em mulheres, apontado como principal fenômeno no atual momento da epidemia ${ }^{14}$.

0 impacto do diagnóstico tardio torna preocupante 0 impacto social e de letalidade, num processo cada vez maior de pauperização e de interiorização ${ }^{6}{ }^{619} \mathrm{em}$ áreas de isolamento geográfico e de características peculiares étnico-culturais ${ }^{32914}$ como na Cidade de Manaus. Os resultados apresentados nesse artigo nos reportam a uma realidade de diagnóstico e tratamento tardio entre os casos estudados, denotando a falta de acesso ao diagnóstico precoce nos serviços de saúde.

0 relatório de progresso de cumprimento de metas do Programa Nacional de DST/Aids do Brasil, na Sessão Especial da Assembléia Geral das Nações Unidas - UNGASS em 2008, destacou que o diagnóstico tardio da infecção pelo HIV/Aids no país ainda é significante, correspondendo a 43,7\%, e o óbito, ainda no início do tratamento alcança $28,7 \%$. Em concordância com os achados do estudo, nesse mesmo relatório, relacionado ainda ao diagnóstico tardio, a região Norte se destacou negativamente no país ao apresentar o pior índice com 53,3\%, enquanto a Região Sul apresentou o menor valor, $40,8 \%{ }^{11}$.

No período total estudado (1986 a 2000), a regularidade ao tratamento dos casos de Aids na Cidade de Manaus evoluiu relativamente de forma satisfatória, necessitando, ainda, de maiores investimentos no serviço de atendimento a esses casos. A redução do óbito ainda no início do tratamento, possivelmente, indica algum grau de melhoria na qualidade da assistência prestada e da atenção às DST/Aids. A introdução da terapia antiretroviral e dos exames laboratoriais de monitoramento terapêutico, quantificação da carga viral e de células CD4+, a partir de 1996 influenciaram na redução dos óbitos e aumento da sobrevida dos casos de infecção pelo HIV/Aids na cidade.

A respeito da mortalidade por Aids no Brasil, Fonseca e Barreira $^{5}$, em estudo descritivo da mortalidade por AIDS no período de 1986 a 1999, registraram 113 mil óbitos de indivíduos em 15 anos ou mais. Entre os maiores de 13 anos de idade, a mortalidade proporcional ocorre com tendência de redução, chegando aos 19,9\% em 1999. Ao longo do período de 1996 a 2006, evidenciou-se redução no coeficiente de mortalidade na região Sudeste, estabilização no Sul e Centro-Oeste e aumento no Norte e Nordeste ${ }^{17}$.

Espacialmente, esses dados apresentam evidências de que a infecção pelo HIV/Aids na Cidade de Manaus evoluiu com distintos padrões de distribuição, ampliação e intensificação epidêmica progressiva, cujas características definiram sua consolidação nos bairros inicialmente afetados ainda na emergência epidêmica, difundindo-se posteriormente para os demais espaços receptivos da cidade. 0 deslocamento nessa área central da Cidade de Manaus acontece, sobretudo, a partir Porto de Manaus e do terminal de transporte coletivo municipal. 
Entre os fatores limitantes dos dados analisados, o método empregado no estudo utilizou dados coletados a partir dos registros de casos nos prontuários, os quais, por sua vez, estavam incompletos e sedimentados na percepção dos profissionais de saúde que os preenchiam ${ }^{1}$. A ausência de registros referentes à clínica, os comportamentais e geográficos refuta outros achados no estudo que corroborariam na descrição do padrão epidêmico e espacial de Manaus. Apesar das limitações dos estudos seccionais, Rodrigues-Júnior e Castilho ${ }^{14}$ afirmam que estudos descritivos sobre a epidemia de Aids podem ter abordagem geográfica, temporal e populacional, para caracterizar a mobilidade, a tendência e a vulnerabilidade, na observação dos fenômenos de transição do perfil epidemiológico. Afinal, analisar a epidemia de Aids por meio dos casos é estudar o passado histórico da epidemia, e construir uma imagem que permita observar trajetórias e processos, pois não é possível apreender todas as faces do acontecido ${ }^{1}$.

0 método aplicado no presente estudo permitiu descrever os padrões epidêmicos e espaciais da distribuição da infecção pelo HIV/Aids em Manaus no período epidêmico, de 1986 a 2000, apresentando além de características epidemiológicas, as clínicas e espaciais. Os dados foram usados como fonte de conhecimento para o planejamento de ações específicas e de estratégias locais para o enfrentamento do problema de saúde pública. A perspectiva é utilizar esses dados para o monitoramento da difusão da infecção pelo HIV/Aids em Manaus e estimular a análise dos dados de 2001 a 2010.

\section{REFERÊNCIAS}

1. Bastos MSCBO, Latorre MRD, Waldman EA. Tendência da epidemia de AIDS em usuários de drogas injetáveis no Município de São Paulo de 1985 a 1997. Revista Brasileira de Epidemiologia 4: 178-190, 2001.

2. Benzaken A, Sardinha JC, Casado R. Aids no Amazonas: retrospectiva histórica e situação atual. In: Rojas LBI, Toledo LM (eds) Espaço e Doença Um Olhar Sobre o Amazonas, Fundação Oswaldo Cruz, Rio de Janeiro, p. II.10.1-II.10.5, 1998.

3. Brito AM, Castilho EA, Szwarcwald CL. AIDS e infecção pelo HIV no Brasil: uma epidemia multifacetada. Revista da Sociedade Brasileira de Medicina Tropical 34: 207-217, 2000.
4. Carneiro Filho A. Manaus: fortaleza extrativismo-cidade, uma história da dinâmica urbana. In: Rojas LBI, Toledo LM (eds) Espaço e Doença Um Olhar Sobre o Amazonas, Fundação Oswaldo Cruz, Rio de Janeiro, p. I.6.1-I.6.5, 1998.

5. Fonseca MGP, Barreira D. A mortalidade da aids no país segundo sua distribuição geográfica. Boletim Epidemiológico de Aids 3: 43-49, 2000.

6. Fonseca MGP, Bastos FI. Twenty-five years of the AIDS epidemic in Brazil: principal epidemiological findings, 1980-2005. Cadernos de Saúde Pública, 23:S333-S344, 2007.

7. Fonseca MGP, Travassos C, Bastos FI, Silva NV, Szwarcwald CL. Distribuição social da aids no Brasil, segundo participação no mercado de trabalho, ocupação e status sócio-econômico dos casos de 1987 a 1998. Cadernos de Saúde Pública; 19: 1351-1363, 2003.

8. Fundação de Medicina Tropical do Amazonas. Coordenação Estadual do Programa de DST/Aids do Amazonas. Boletim Epidemiológico 1: 1-20, 2006.

9. Grangeiro A, Ferraz D, Barbosa R, Barreira D, Veras MASM, Vilela W, Veloso JC, Nilo A. UNGASS-HIV/AIDS: balanço da resposta brasileira, 2001-2005. Revista de Saúde Pública 40 (supl): 5-8, 2006.

10. Instituto Brasileiro de Geografia e Estatística. Base Territorial Censo 2000: Mapeamento Censitário, Atividades e Conceitos Básicos. Brasil: Instituto brasileiro de Geografia e Estatística, 2000; http://www.ibge.gov.Br/dados. (acessado em 20/Jul/2008).

11. Joint United Nations Program on HIV/AIDS. UNGASS 2008: country progress reports, 2008; http://www.unaids.org. (acessado em 14/Mai/2008).

12. Mann J, Tarantola D. AIDS in the World II. New York/Oxford: Oxford University Press, 1996

13. Ministério da Saúde. Coordenação Nacional de DST e Aids. Aids no Brasil: um esforço conjunto governo-sociedade. Brasília, p. 11-18, 1998.

14. Rodrigues-Júnior AL, Castilho EA. A epidemia de AIDS no Brasil, 1991-2000: descrição espaço- temporal. Revista da Sociedade Brasileira de Medicina Tropical 37: 312-317, 2004

15. Sabroza PC, Toledo LM, Osanai CH. A organização do espaço e os processos endêmico-epidêmicos. In : Leal MC, Sabroza PC, Rodrigues RH, Buss PM (eds) Saúde, Ambiente e Desenvolvimento. HUCITEC/ABRASCO, São Paulo/Rio de Janeiro, p. 57-77, 1992

16. Secretaria de Vigilância em Saúde. Programa Nacional de DST e Aids. Critérios de definição de casos de aids em adultos e crianças, Ministério da Saúde, Brasília, p. $17-21,2003$

17. Secretaria de Vigilância em Saúde. Programa Nacional de DST e Aids. Boletim Epidemiológico Aids e DST, Ministério da Saúde, Brasília 5: 1- 46, 2007.

18. Silva Neto AL. Estudo da associação entre tuberculose e a infecção pelo HIV no município de Manaus, Amazonas. Dissertação de Mestrado, Escola Nacional de Saúde Pública, Fundação Oswaldo Cruz, Rio de Janeiro, RJ, 2001.

19. Szwarcwald CL, Bastos FI, Esteves MAP, Andrade CLT. A disseminação da epidemia da AIDS no Brasil, no período de 1987-1996: uma análise espacial. Cadernos de Saúde Pública 16(supl 1):S7-\$19, 2000. 